\title{
Les scènes imaginaires de la réécriture
}

\section{Carole Egger}

\section{(2) OpenEdition}

\section{Journals}

Édition électronique

URL : https://journals.openedition.org/cher/1968

DOI : $10.4000 /$ cher.1968

ISSN : 2803-5992

\section{Éditeur}

Presses universitaires de Strasbourg

\section{Édition imprimée}

Date de publication : 29 mai 2018

Pagination : 11-19

ISBN : 979-10-344-0019-5

ISSN : 1968-035X

\section{Référence électronique}

Carole Egger, «Les scènes imaginaires de la réécriture », reCHERches [En ligne], 20 | 2018, mis en ligne le 01 décembre 2021, consulté le 02 décembre 2021. URL : http://journals.openedition.org/cher/1968 ; DOI : https://doi.org/10.4000/cher.1968

\section{c) (i) (2) (-)}

Ce(tte) œuvre est mise à disposition selon les termes de la Licence Creative Commons Attribution Pas d'Utilisation Commerciale - Partage dans les Mêmes Conditions 4.0 International. 


\title{
Les scènes imaginaires de la réécriture
}

\author{
Carole EgGeR ${ }^{1}$
}

$\mathrm{L}$ a réflexion que je propose ici se déploie essentiellement dans le champ du théâtre et des arts de la scène occidentale, et plus particulièrement dans le monde hispanique - qui constitue mon domaine de recherche privilégié -, même si je m'attache parfois à extrapoler certaines conclusions afin de les adapter à la multiplicité des formes que peut prendre le processus de réécriture au sens large, dont celle de la traduction et de l'adaptation. Après quelques considérations, à la fois théoriques et pratiques, concernant les fondements des différentes approches de la réécriture, je m’intéresserai plus spécifiquement à la question de la traduction, comme forme singulière, et peut-être majeure, du processus.

L'acte de naissance du théâtre dans la Grèce Antique est scellé par la réécriture. Depuis l'origine, les dramaturges réécrivent, réélaborent, traduisent et adaptent des matériaux identifiables par l'imaginaire collectif grâce aux rhapsodes qui, comme les trouvères et les troubadours au moyen âge, los juglares en Espagne, allaient de ville en ville réciter, probablement en les mimant, les poèmes épiques, en particulier les poèmes d'Homère. Les mystères médiévaux ne sont que des récréations vivantes de scènes bibliques et lorsque le monde occidental redécouvre le théâtre au sortir du Moyen Âge, thèmes traditionnels ou folkloriques, mythes et tragédies antiques fourniront à nouveau matière à réécriture.

Au théâtre, à un premier niveau, c'est sans doute dans la contrainte due à la présence d'un public qu'il faut chercher les raisons de cette réélaboration sans cesse renouvelée d'éléments épiques, mythiques, historiques, folkloriques,

1 Professeur de langue et littérature espagnoles à l'Institut d'études romanes. Faculté des Langues, Université de Strasbourg.

Suite à un colloque qui s'est tenu à l'Université de Waseda à Tokyo en octobre 2016, cet article a été publié en japonais dans『越境する 翻訳・翻案・異文化交流』 (Dépasser les frontières: traductions, adaptations et communications interculturelles), Musée du Théâtre, Université Waseda, mars 2017. 
fictionnels, etc., qui fait de la réécriture - dont la traduction et l'adaptation ne sont que des variantes - une des spécificités du genre théâtral. Pour qu'une poétique théâtrale puisse atteindre son objectif, qui demeure toujours en dernier ressort le plaisir du public, il convient que ce dernier possède les clés d'accès à l'œuvre. La réécriture est sans doute un des principaux procédés de ce «pacte dramatique implicite» que toute pièce doit respecter afin, comme dirait Mnouchkine, que «le public puisse jouer» (Mnouchkine 1975).

Les modalités de la réécriture se sont largement diversifiées dans les productions contemporaines. Elles dépendent à la fois du matériau d'origine (poésie, théâtre, roman, chronique historique, texte biblique, mythe en tous genres...), du traitement que l'auteur se propose de lui appliquer (parodie, pastiche, réinvention, rénovation, réélaboration, réactualisation...) en fonction de l'objectif qu'il poursuit (simple recréation, questionnement de fond, réappropriation de soi, du passé, de l'histoire...). Car la réécriture se déploie aussi bien dans le champ de la sémantique que dans celui de la linguistique, dans le champ de la stylistique que dans celui de la poétique.

Les approches théoriques parfois diffèrent selon que l'on privilégie la dimension littéraire de la réécriture, qui suppose un modèle original à partir duquel s'engage le processus - le mythe, Médée, Antigone, Don Juan, etc. -, ou bien la dimension linguistique, qui s'attache aux reprises, approximations successives et différents états de l'élaboration d'un texte.

Ainsi a-t-on interrogé la réécriture d'un point de vue formel: quels aménagements impose le passage à la scène ou la transgression d'un genre? Quelles coupes, quels raccourcis, quelles modifications induit, par exemple au théâtre, la mise en bouche d'un texte? Quelles contraintes l'oralité et la scène induisent-elles dans les tracés syntaxiques des répliques, leur extension, leur rythme, leur intonation? Quels obstacles linguistiques, phonétiques, lexicaux, syntaxiques franchit-on en transgressant les frontières d'un genre ou d'une époque? Autant de questions qu'il est loisible de poser d'un genre à un autre, d'une poétique à une autre, d'un dramaturge à l'autre.

La réécriture implique, souvent, une transgression de genre: du roman au théâtre, du théâtre au cinéma, de la poésie au théâtre, etc., mais pas toujours, et parfois une même source (par exemple le mythe de don Juan) fait l'objet de multiples réécritures au sein d'un (le théâtre) ou de plusieurs genres (cinéma, roman, musique, peinture, poésie, danse, etc.).

L'examen du processus de réécriture, protéiforme et proliférant, et pas seulement dans le champ de la littérature, peut aussi s'attacher à sa dimension plus idéologique que formelle. Il est alors possible d'interroger la réécriture, et ses différentes modalités, en tant que force de contestation, ou bien en tant que processus susceptible de mettre en crise un système générique, un système de pensée et au-delà, peut-être également un rapport au monde.

Dans son ouvrage Dramaturgie de textes narratifs (Sinisterra 2003), le dramaturge et théoricien espagnol José Sanchis Sinisterra propose de privilégier 
dans le processus de réécriture, non le champ de ce qu'il appelle «la dramaturgie fabulaire ", c'est-à-dire celle qui renvoie aux articulations de l'histoire structurant l'action (dans une perspective aristotélicienne) mais «la dramaturgie discursive», c'est-à-dire renvoyant au contexte idéologique, dramatique et scénique le plus susceptible d'intensifier l'expérience du récepteur (lecteur ou auditeur):

L'effet produit par un texte sur le lecteur ne dépend pas tant de la fable, ne dépend pas tant de l'histoire qui y est racontée mais bien plutôt de la manière dont le narrateur la raconte ${ }^{2}$. (Sinisterra 2003: 61)

En se situant systématiquement dans une perspective pluridisciplinaire, il s'agit d'explorer un contenu et lui redonner vie dans un nouveau contexte où la question des frontières, d'un genre à l'autre, d'une aire géographique à une autre, d'un imaginaire culturel à un autre, est en mesure de potentialiser le propos.

José Sanchis Sinisterra ne cherche pas à domestiquer les textes narratifs pour les soumettre aux contraintes du genre théâtral. Ce qu'il traque, c'est davantage une théâtralité différente, qui défie et questionne la narration, une théâtralité nouvelle et contemporaine, spécifique à chaque auteur, comme peuvent l'être celles de Beckett, de Pinter ou encore de Tchékhov. Pour Sanchis Sinisterra, le théâtre doit puiser dans l'énorme richesse formelle de la narration car ce transfert générique offre un champ particulièrement étendu à la réflexion et à la dramaturgie contemporaine. Filtrer la dramaturgie à l'aune de l'épique devient ainsi, parallèlement aux recherches de l'auteur dans le champ des sciences dures appliquées au théâtre (mathématiques, physique, linguistique) ou de l'art (minimalisme, danse-théâtre), une manière de remettre en question les structures dramatiques conventionnelles pour renouveler la forme dramatique. Pour que toute rénovation théâtrale ne soit plus condamnée, comme c'est souvent le cas, à n'être qu'un dépassement des formes dramatiques précédentes, Sinisterra propose une voie nouvelle, celle qui consiste à prendre en compte ce qui se passe dans d'autres champs de la littérature et de l'art et même dans le champ des sciences dures ou encore de la médecine. La scène devient ainsi emblématique d'un dialogue inter-générique et interdisciplinaire susceptible de mieux approcher la complexité du monde.

La réécriture subit également les contraintes du temps et du contexte. En Espagne, face au désert culturel franquiste par exemple, nombre de dramaturges au cours du vingtième siècle ont en outre consciemment cherché ailleurs (autres lieux, autres époques) des sources d'inspiration ou se sont servis de cet instrument pour revisiter l'Histoire. L'intertextualité servait alors fréquemment de biais pour déjouer les pièges de la censure.

De même, passée la période de la dictature, où le théâtre du Siècle d'or faisait l'objet d'un certain ostracisme de la part des milieux intellectuels et artistiques - car il était alors généralement considéré comme réactionnaire -, la réécriture des classiques connaît aujourd'hui un formidable essor sous la double impulsion

2 C'est moi qui traduis. 
de la Compañía Nacional de Teatro Clásico et du Festival d'Almagro, né en 1978. Beaucoup d'auteurs contemporains parmi les plus reconnus, comme Juan Mayorga par exemple, s'adonnent ainsi à la réécriture des pièces du répertoire.

Au niveau théorique, la critique génétique est née dans les années 1970 précisément de cette volonté d'explorer cette part du texte qui appartient à d'autres textes et en réaction au structuralisme et aux théories du texte qui envisageaient le texte de manière isolée, dans sa singularité. Aujourd'hui, dans le domaine du théâtre, la critique s'étend jusqu'à intégrer des éléments qui ne relèvent pas du langage écrit. Ainsi la genèse du spectacle théâtral ne travaille plus sur les différents états et transformations d'un texte - «les brouillons textuels» - mais sur les brouillons visuels que constituent les cahiers des mises en scène successives, les enregistrements visuels, les notes de répétitions, etc., soit «les brouillons scéniques».

La révolution symboliste de la fin du $\mathrm{XIX}^{\mathrm{e}}$ siècle avait contribué à remettre sur le devant de la scène littéraire la question de la fragilité des frontières entre l'illusion et la réalité. Dès lors le questionnement métalittéraire (ou métathéâtral, ou métapictural, etc.) est redevenu central au cours du $\mathrm{xx}^{\mathrm{e}}$ siècle. Rien d'étonnant dès lors que la réécriture non seulement a ainsi constitué au cours des dernières décennies un des axes privilégiés de la recherche en sciences humaines, et en particulier, dans le domaine de la littérature et des arts du spectacle, mais a même constitué la caractéristique centrale d'œuvres majeures et universelles comme celle de Jorge Luis Borges par exemple (Lafon 1990).

Dans le domaine de la recherche, de nombreux colloques et publications ont ainsi exploré les frontières, souvent très floues, entre création et recréation, entre traduction et adaptation, entre adaptation et réécriture, et ont examiné le potentiel de créativité que constitue la recherche de nouveaux moyens d'expression comme de nouvelles formes de narration, d'écriture scénique ou cinématographique.

Au théâtre, la mise en scène-illustration d'un texte a concouru à l'invention de potentialités scéniques utilisant de plus en plus les nouvelles technologies, et l'intermédialité devient ainsi centrale dans de nombreuses propositions dramaturgiques actuelles.

De même, ont été explorés tous les procédés conduisant à la réélaboration formelle d'une œuvre: découpage, réduction ou compression, ellipses, focalisations, flash-back... ou bien conduisant à la reformulation sémantique: transformations, actualisations, détournements...

D’autres programmes ont travaillé le rapport entre tradition et modernité et ont centré la recherche sur les limites entre conservatisme et innovation.

Si les échos et les jeux intertextuels participent de plus en plus des fictions en tous genres (roman, théâtre, poésie) et infiltrent même tous les domaines de la pratique artistique (peinture, danse, musique), c'est aussi sans doute parce que tous les champs de la connaissance n'ont cessé d'explorer, tout au long $\mathrm{du} \mathrm{Xx}^{\mathrm{e}}$ siècle et au début $\mathrm{du} \mathrm{XXI}^{\mathrm{e}}$, ce que le moi doit à l'Autre, l'Autre 
entendu désormais non plus seulement comme concept psychanalytique mais dans un sens plus largement anthropologique. L'originalité, qui avait inauguré la modernité, n'occupe plus le devant de la scène. Les œuvres se construisent sciemment, explicitement, dans l'ombre des grandes figures tutélaires car leurs auteurs, conscients de la part héritée de leur imaginaire et de leur créativité, assument de plus en plus ouvertement leur héritage. Mais il ne s'agit plus, comme pour l'humanisme de la Renaissance et du Xvir ${ }^{\mathrm{e}}$ siècle redécouvrant l'Antiquité gréco-latine, d'une imitation des anciens, d'une inscription délibérée dans une lignée d'œuvres faisant autorité. La valeur de l'œuvre actuelle ne se mesure plus à sa capacité à redonner vie à l'œuvre ancienne en l'enrichissant dans un style propre mais à jouer à l'envi du concept de dialogisme - inauguré par Bakhtine - et de celui d'intertextualité (Julia Kristeva) ainsi que de leurs dérivés (interthéâtralité, interpicturalité, intericônicité, etc.). Avec l'avènement d'une nouvelle ère du doute et de l'incertitude - une ère «néo-baroque» de ce point de vue, qualificatif qui a souvent servi à définir quelques-unes des caractéristiques de la postmodernité -, il y a là un véritable changement de paradigme.

Dans tous les champs de la littérature et des arts du spectacle, les formes de réécriture sont tellement nombreuses que d'aucuns en sont arrivés à établir qu'il y avait recouvrement du champ de la littérature et de celui de la réécriture. Autrement dit, toute la littérature ne serait que traduction, adaptation ou réécriture, à tel point qu'on peut se demander si les fondements du processus de réécriture ne seraient pas à inscrire et à explorer, là encore, dans une perspective plus largement anthropologique.

La porosité des frontières, d'une langue à l'autre, d'un espace culturel et temporel à un autre, rend compte de la complexité mais aussi de la permanence du processus. L'histoire de l'Humanité s'est racontée depuis la nuit des temps à travers des histoires, des contes, des fables, des chroniques, etc., que l'on réécrit, retranscrit, adapte comme s'il s'agissait de sceller, au-delà des instrumentalisations contingentes, à travers le temps et l'espace, la continuité de l'imaginaire.

La fortune de la réécriture est par ailleurs tributaire de la faille radicale existante entre «les mots et les choses» (Foucault 1966); c'est parce que le langage est toujours conventionnel, fondamentalement dénotatif, qu'il n'y a jamais recouvrement entre le mot - et encore moins la phrase - et la chose, ou la réalité. L'inachèvement de toute écriture est inscrit dans cette faille, de même que la réécriture est la façon de «vingt fois sur le métier» remettre «son ouvrage $»^{3}$ afin de tendre vers une forme plus adaptée (le mieux dire) ou vers un

3 Selon la célèbre formule de Boileau [Boileau (1669-1674), Chant Premier v. 171-174]. La citation exacte est:

Hâtez-vous lentement, et sans perdre courage,

Vingt fois sur le métier remettez votre ouvrage

Polissez-le sans cesse, et le repolissez,

Ajoutez quelquefois, et souvent effacez. 
contenu plus précis (le dire vrai ou le mieux «traduire»). Jacqueline AuthierRevuz l'exprime de façon transparente:

C'est cela qui est l'expérience fondamentale de l'être humain, à savoir que la langue «rate» le monde et que c'est malgré tout la seule manière qu'il ait de l'attraper, - chose quand même tragique dans l'expérience humaine!

Dans le champ linguistique, le discours théorique a ainsi peu à peu imposé puis érigé en évidence le fait qu'il n'existe pas d'écriture sans réécriture, pas de phrase sans paraphrase, ni de formulation sans reformulation. Almuth Gresillon évoque la dimension métalangagière de la réécriture qui rappelle que la distance du langage vis-à-vis de lui-même est une dimension proprement humaine.

C'est un privilège de l'être humain que ce qui lui est le plus intime, le plus fondateur, ce qui fait de lui un sujet, soit en même temps ce qui lui est extérieur, qui lui vient de l'extérieur, qui lui reste extérieur. C'est-à-dire que nous sommes à distance du langage, mais pas sur le mode où on est à distance d'un instrument. C'est bien ça qui en fait la spécificité, c'est-à-dire que finalement on est à distance de quelque chose qui crée une distance à l'intérieur, en nous. (Boré C., DoquetLacoste C. 2004)

Il est possible que l'acte fondateur du théâtre au sortir du moyen âge soit lié à cette distance de soi à soi d'un individu des Temps Modernes qui ne porte plus le discours de la collectivité mais son propre discours. La distance nouvelle qui se crée alors entre scène et salle serait ainsi tributaire de cette énonciation scindée entre un individu qui porte la parole, et le même individu qui la reçoit. Jacqueline Authier-Revuz rappelle que

Bakhtine pose - [et dit-elle] je suis profondément d'accord avec lui - qu'à tout moment on est récepteur de sa propre parole et de son propre écrit, et par conséquent que, en tant qu'énonciateurs, nous sommes en permanence «faillés » par notre propre écoute, intimement. (Boré C., Doquet-Lacoste C. 2004)

La langue fonctionne ainsi de manière clivante pour l'énonciateur, lequel devient progressivement à la fois producteur et récepteur de sa propre parole. Les rapports complexes entre les premières formes de discours et l'émergence d'un individu des Temps Modernes, dont on ne peut que constater qu'elle est concomitante de l'émergence de formes théâtrales mettant à distance la scène et la salle, sont encore à explorer. C'est en effet l'époque où l'occident construit ses premières salles de spectacle. Après la découverte de la perspective dans l'Italie du Quattrocento qui suppose un processus d'abstraction - puisque l'appréhension du monde passe désormais par une illusion tridimensionnelle qui fait que le Réel est autant constitué par ce que je vois que par ce que je ne vois pas - le théâtre en effet se dote de lieux spécifiques à la représentation où acteurs et spectateurs seront désormais parfaitement distingués, mettant ainsi à distance la langue de l'Autre, redoublant ainsi le dialogisme de la langue. La réécriture pourrait ici se concevoir comme inhérente au langage et au clivage de la conscience entre soi et l'autre, entre le Même et l'autre et "L'autre du même», ces deux dernières expressions étant respectivement le titre d'un ouvrage désormais classique de Vincent Descombes - où le philosophe montre qu'après 1960, la philosophie ne 
considère plus le monde comme soumis à un unique Ego - et le titre d'un court article de Gérard Genette où le théoricien de la littérature montre qu'on ne peut varier sans répéter ni répéter sans varier.

Borges en est tellement convaincu que pour lui, qui passe indifféremment de la traduction de l'Autre à la traduction de soi, "création et traduction sont des activités équivalentes - aussi tragiques, aussi futiles - (...)» - selon la formulation de Michel Lafon - et «toutes les œuvres sont l'œuvre d'un seul auteur qui est intemporel et anonyme» (Borges 1957: 47).

Proust, l'avait précédé qui, déjà au début $\mathrm{du} \mathrm{xx}^{\mathrm{e}}$ siècle, remarquait que chaque auteur était contraint par un creuset d'œuvres préexistant à son écriture:

Ainsi j'étais déjà arrivé à cette conclusion que nous ne sommes nullement libres devant l'œuvre d'art que nous ne la faisons pas à notre gré, mais, que, préexistant à nous, nous devons, à la fois parce qu'elle est nécessaire et cachée, et comme nous ferions pour une loi de la nature, la découvrir. (Proust 1982: 881)

L'auteur de La recherche avait également avancé l'idée que la tâche principale de tout écrivain était de traduire:

Je m'apercevais que ce livre essentiel, le seul livre vrai, un grand écrivain n'a pas, dans le sens courant, à l'inventer, puisqu'il existe déjà en chacun de nous, mais à le traduire. Le devoir et la tâche d'un écrivain sont ceux d'un traducteur. (Proust 1982: 890)

Borges, qui a lui-même longuement pratiqué la traduction, a ainsi inauguré une nouvelle ère dans ce domaine, en se moquant ouvertement de la tyrannie de la paternité littéraire et en multipliant les jeux intra et intertextuels, considérant que la littérature se moque des nationalités et des époques. Ainsi pour lui, la traduction ne doit pas consister pas en un simple passage d'une langue à une autre mais en un véritable processus de transfusion où il s'agit de rendre compte du répertoire de procédés poétiques et narratifs, de modèles et de méthodes, possédé par chaque langue.

Dans son sillage, et pour relever quelques-unes des conceptions du rapport traduction/adaptation/réécriture qui me paraissent les plus intéressantes, Juan Mayorga propose de considérer comme des traducteurs tous ceux qui interviennent «dans la problématique transmission d'une œuvre du passé» qu'il s'agisse d'un musicien, qui crée une nouvelle version d'un morceau classique ou d'un commissaire d'exposition qui opère une sélection d'œuvres picturales et les présente «dans un certain espace, dans un certain ordre et sous un certain titre».

Je propose - dit-il - de considérer comme des traducteurs en général (...) tous ceux qui réalisent un déplacement qui est traduction, c'est-à-dire, une opération qui est à la fois conservatrice et créatrice. Du mouvement de la traduction, qui inclut autant la continuité (la transmission) que l'interruption (la rupture), le "toujours identique» de la répétition infinie n'est pas de mise, comme n'est pas de mise la prétention de parler un langage adamique, primordial. À la limite - dans la mesure où on ne traduit pas seulement des paroles mais aussi des idées, des formes, des gestes - chaque création peut être considérée comme une traduction. Parce que dans chaque œuvre sont sauvegardées d'autres œuvres qui 
l'ont précédée, comme dans chaque style est sauvegardé le style qui est cité et ceux qui sont rejetés. Finalement, l'histoire de la culture peut être vue comme une incessante migration de pays en pays, d'époque en époque, d'art en art: comme une infinie traduction. (Mayorga 2016: 147)

Juan Mayorga est sensible à l'inachèvement constitutif de toute œuvre. Il s'inscrit, en ce sens, dans une conception benjaminienne du temps où l'histoire ne cesse de proposer de nouvelles lectures et de nouvelles interprétations du passé. «Le temps qui me traverse - dit-il - opère une constante modification et réécriture de mes textes.» Le dramaturge a de fait multiplié les adaptations d'œuvres aussi bien classiques (Euripide, Calderón, Lope de Vega, Shakespeare, etc.) que plus modernes (Dostoïevski, Ibsen, Tchekhov, Valle-Inclán, Kafka, Durrenmatt, Primo Levi, etc.). Pour le dramaturge contemporain, adapter un texte, c'est le traduire, l'emmener d'un temps vers un autre, c'est enrichir la conscience du temps du spectateur.

Sa conception de la traduction s'inspire par ailleurs de celle du philosophe judéo-allemand Walter Benjamin (1892-1940), sur l'œuvre duquel il a écrit une thèse de doctorat. Pour Benjamin, toutes les langues sont apparentées et entretiennent entre elles un rapport intime. Le langage humain par ailleurs est muet en ce sens qu'il n'épuise jamais la réalité du monde. Le philosophe qualifie de vrai, de pur langage cet espace inaudible où les langues se rejoignent et qu'aucune langue ne peut faire advenir. "La tâche du traducteur est de faire mûrir, dans la traduction, la semence du pur langage», autrement dit la traduction permet de représenter en germece langage supérieur apte à laisser entrevoir l'ineffable, l'intangible, l'incommunicable. Écoutons Mayorga parler de cette conception à la fois romantique et messianique de la traduction :

La meilleure traduction entre deux langues, selon Benjamin, est celle capable de faire que ces deux langues se reconnaissent comme des fragments d'un langage supérieur. En ce sens, dans la traduction est représenté un langage pur, la langue de la vérité, la fin même de la philosophie. Chaque traduction est pour Benjamin non seulement une forme de survivance de l'œuvre originale, mais aussi la représentation du mouvement infini des langues vers un langage vrai. L'espoir de ce langage absolument autre réside donc entre les lignes, dans l'espace de ce qui n'est encore écrit dans aucune langue. (Mayorga 2016: 148)

On pourrait concevoir cette émergence du pur langage comme l'objectif ultime et inconscient de cet inlassable et dynamique travail de traduction/adaptation/ réécriture, qui demeure une véritable marque de fabrique de l'histoire de l'humanité. Peut-être s'agit-il là de la scène imaginaire la plus riche de toute réécriture. 


\section{Bibliographie}

Boileau N., 1998, Art poétique (1669-1674), Paris, Flammarion.

Borges J.L., 1957, «Tlön, Uqbar, Orbis, Tertius» in : Fictions, Paris, Gallimard.

Boré C., Doquet-Lacoste C., 2004, «La réécriture questions théoriques», Le français aujourd'hui, n 144, p. 9-17, https://www.cairn.info/revue-le-francaisaujourd-hui-2004-1-page-9.htm

Foucault M., 1966, Les mots et les choses: une archéologie des sciences humaines, Paris, Gallimard.

Lafon M., 1990, Borges ou la réécriture, Paris, Seuil, coll. «Poétique».

Mayorga J., 2016, Elipses, Segovia, La uŇa RoTa.

Mnouchkine A., 1975, Théâtre Public 5.6, juin/juil./août.

Proust M., [1927] 1982, Le temps retrouvé, Paris, Gallimard, Bibliothèque de la Pléiade.

Sinisterra J.S., 2003, Dramaturgia de textos narrativos, Ciudad Real, Naque. 